\title{
Current Trends in Treating Acanthamoeba Keratitis: A Brief Narrative Review
}

\author{
Mauro Salducci ${ }^{1}$ \\ ${ }^{1}$ Department of Ophthalmology, Sapienza University of Rome (Italy)
}

\begin{abstract}
The author, after examining the historical evolution of scientific knowledge and treatment of severe Acanthamoebic keratitis, presents this brief review on the treatment of this serious eye disease, relatively frequent in patients with corneal contact lenses. Therapy of Acanthamoeba keratitis is always very long and demanding. Its management requires adequate experience because it is not always easy to evaluate the response to treatment and complications can be very serious and difficult to manage. Resistance to therapy can also occur during treatment and must be distinguished from drug-induced toxicity. In cases where no improvement is obtained with maximum medical therapy, it is advisable to repeat the corneal sampling and proceed to new laboratory tests for Acanthamoeba, bacteria and fungi. Prevention, which always remains of fundamental importance, is practically based on avoiding contact of the corneal lens with contaminated water, since this Acanthamoeba has a ubiquitous diffusion. It is therefore recommended to always avoid the use of corneal contact lenses in the pool or in the shower, not to wash them under running tap water and to frequently replace the relative container of these lenses.
\end{abstract}

Key Words: Acanthamoeba Keratitis, Corneal ulcer, Contact lenses.

How to Cite this Article: Mauro. Current Trends in Treating Acanthamoeba Keratitis: A Brief Narrative Review. Pak J Ophthalmol. 2020; 36 (4): 451-455.

Doi: https://doi.org/10.36351/pjo.v36i4.1068

\section{INTRODUCTION}

Since 1973, it has been recognized that Acanthamoeba microorganism, which is part of the amoeba family, causes severe keratitis which can lead to blindness. The characteristic symptoms include severe eye pain, paracentral annular stromal infiltrates, epithelial ulcers and resistance to most antibiotics. ${ }^{1}$ Number of recognized cases of Acanthamoeba keratitis has increased steadily in recent years for various reasons. These include increased awareness of ophthalmologists, increase in the number of contact

Correspondence: Mauro Salducci

Department of Ophthalmology,

Sapienza University of Rome (Italy)

Email:mauro.salducci@uniromal.it

Received: May 18, 2020

Accepted: July 27, 2020 lens wearers, lack of proper care in contact lens hygiene and availability of rapid diagnostic tests that allow confirmation of Acanthamoeba infection.

Acanthamoeba is a ubiquitous microorganism that can be isolated from a wide variety of environments, especially from ponds, swimming pools, reservoirs, sea, hot tubs, salt water, bottled water and saline solutions for contact lenses. As a pathogen, Acanthamoeba can cause a form of chronic granulomatous encephalitis and skin amoebiasis in immunocompromised individuals, as well as a severe form of chronic keratitis in the healthy population. ${ }^{2}$ Corneal infection is often associated with the use of contact lenses, which in fact represents the most important risk factor. Some studies have shown that more than $80 \%$ of cases of Acanthamoeba keratitis occur in contact lens wearers. ${ }^{3}$

Acanthamoeba exists in two forms, quiescent cyst and active trophozoite form. Under unfavorable 
conditions, trophozoites are encysted. The cyst has a double wall containing cellulose with a diameter of $10-25 \mu \mathrm{m}$; it is extremely resistant to extreme conditions, such as changes in osmolarity, $\mathrm{pH}$, drying, freezing or antimicrobial chemical agents. Although the clinical symptoms of Acanthamoeba keratitis can be controlled by various pharmacological agents, parasites can encyst in the corneal stroma and remain in a quiescent form. A corneal transplant can thus activate the quiescent cysts in the limbus and cause a resurgence of keratitis. ${ }^{4}$

\section{BRIEF REVIEW}

The first case of Acanthamoeba keratitis was described by Jones in 1975. Only 10 cases of Acanthamoeba keratitis were reported between 1975 and 1981. Since 1981 the number of cases has increased gradually and more than 100 cases have been reported in the United States in recent years. ${ }^{5}$ In Great Britain, about 400 cases of Acanthamoeba keratitis have been reported since 1977, but the real incidence of Acanthamoeba keratitis is unknown. ${ }^{6}$ More than 750 cases have been diagnosed worldwide. However authoritative authors suggest that the incidence of Acanthamoeba keratitis can be 1 per 10,000 contact lens wearers per year. Theoretically speaking, as there are $1,800,000$ contact lens wearers in Italy, infections with Acanthamoeba would be around 180 per year. $^{7}$

Acanthamoeba Keratitis occurs in young and immunocompetent individuals, most of whom are contact lens wearers and it equally affects men and women. The pathology occurs more frequently unilaterally, although bilateral cases have also been observed and the main risk factors that seem to be implicated in the onset of Acanthamoeba keratitis are; previous corneal trauma, cleaning of contact lenses with tap water or a solution contaminated with Acanthamoeba and the use of contact lenses. ${ }^{8}$

From the point of view of clinical signs, one of the most important symptoms in case of Acanthamoeba keratitis is severe pain, which is very disproportionate to the clinical aspect, especially in the early stage of infectious process. This is linked to perineural infiltration. Early infection can be confined to epithelium, which shows irregularities. Another important feature is represented by the dendritic form corneal lesions. Late manifestations include stromal infiltrates and a characteristic ring infiltrate. Recurrent corneal erosions, ring infiltrates, corneal abscesses often develop that lead to an incorrect diagnosis of herpetic keratitis, satellite lesions and in severe cases hypopyon, nodular or diffuse anterior scleritis or posterior scleritis. ${ }^{9,10}$ It is therefore essential to obtain a laboratory diagnosis as soon as possible since a delayed therapy can negatively alter the visual outcome. $^{8}$

Corneal scrapings should be performed for staining and culture of epithelial or subepithelial lesions. If epithelial disease is small and stromal infection predominates, a biopsy should be considered followed by staining with Methenamine Silver, Hematoxylin, Eosin and Periodic acid-Schiff (PAS).

Tissues from scraping or biopsy should be transported to the laboratory in saline along with samples taken from the contact lens box and cleaning solutions, if of course they are available. The latter must be inoculated on a lawn of Escherichia coli on non-nourishing Agar, so that the amoebae, which consume E. coli, form identifiable traces. ${ }^{6}$

Electron microscopy and Confocal Microscopy can also be used to identify parasites in corneal tissues, while Phase Contrast Microscopy can be used to identify trophozoites furniture that has a large karyosome and a contractile vacuole. ${ }^{8}$

As for the treatment of this serious keratopathy, the suggested antimicrobials include; Membrane antiseptics (Chlorhexidine and Polyhexamethylene biguanide, PHMB) which inhibit membrane function, Aromatic diamonds (Hexamidine, Pentamidine Isothotate, Propamidine Isothionate) which inhibit DNA synthesis, Aminoglycosides (Neomycin and Paromomycin) which inhibit protein synthesis and Imidazoles (Clotrimazole, Fluconazole, Ketoconazole, Miconazole), which destabilize cell walls. The most commonly used drugs are Propamidine Isothionate (Brolene, not available in Italy but still supplied at the Vatican City pharmacy) and Chlorhexidine Gluconate (Visiodose eye drops), which are easily available in France and Republic of San Marino. ${ }^{11,12,13}$

Therapy combines antimicrobial agents that have different and additive or synergistic mechanisms. Cationic antiseptics have a good general anti-amoebic activity, so, as an initial approach, Chlorhexidine or PHMB is recommended in combination with Neomycin and/or Propamidine Isothionate. If necessary, Imidazole can be added as a third agent. ${ }^{14,15}$

Once the diagnosis is made, the drops are used every hour for 48 hours, the frequency of dropping is 
gradually reduced at night while administration is maintained every hour during the day. As inflammation and infection decreases, the frequency can be reduced within a few weeks to 4 times a day, a dosage, which is then maintained for many months.

The role of corticosteroids in the treatment of Acanthamoebic infection is still controversial. Corticosteroids suppress the host's immune and inflammatory responses and reduce the severity of inflammation. Although topical corticosteroid therapy improves the clinical picture of Acanthamoeba keratitis, both worsening and side effects may occur during the execution of this therapy. ${ }^{16}$

In the early stage of infection, de-epithelialization is effective, if used in combination with anti-amoebic therapy. This procedure appears to increase the penetration of drugs into the cornea and facilitate the removal of pathogenic microorganisms from the lesion.

Corneal cryotherapy has also been used both as a single procedure and in combination with Keratoplasty. Cryotherapy is performed at the time of perforating keratoplasty to destroy and confine parasites outside the transplanted flap. The rationale for this procedure is to eliminate relapses in recipients. ${ }^{17}$ In vitro, trophozoites have been shown to be killed when exposed to temperatures between $-50^{\circ}$ $\mathrm{C}$ and $-130^{\circ} \mathrm{C}$. However, the cysts survive. It is important to note that cryotherapy can cause extensive corneal damage. Cryotherapy therefore represents an ineffective therapeutic procedure in the treatment of Acanthamoeba keratitis. ${ }^{18}$

Lamellar or perforating keratoplasty is recommended in case of progression of the disease, despite the therapeutic regimen, in the event of imminent danger of corneal perforation. ${ }^{19}$ However, the timing of this surgery is still controversial. Most of the published work shows that Keratoplasty is used only in an advanced stage of pathology, that is, when limbus and sclera may already be involved or when corneal perforation has occurred. In fact, the results are often disappointing. The biggest problem is the recurrence of infection and inflammation with cataract complicated by secondary hypertonicity, intraocular inflammation, secondary limbal deficit and rejection. For this reason, some authors suggest early Keratoplasty so as to be sure of eradicating the disease. $^{20}$

Another subject of discussion is the choice of the type of Keratoplasty; Penetrating or Lamellar. Deep lamellar Keratoplasty is indicated in cases where the cornea is not perforated. The advantages of this technique are the lower risk of rejection in these inflamed eyes and a reduced risk of endophthalmitis, being a closed bulb surgical procedure. ${ }^{21}$

The disadvantage is the possibility that infected fibers remain in the residual stroma, a risk that is reduced with the use of deep Keratoplasty techniques, in which the whole stroma is removed. In any case, if a lamellar Keratoplasty fails, a penetrating Keratoplasty can always be used. ${ }^{8}$ If there is an imminent danger of corneal perforation, lamellar Keratoplasty must be done. In this procedure, it is essential that the infected corneal region is entirely included in the donor flap, otherwise the infection can often reappear at the transplant-host interface with drastic consequences.

Therapy of Acanthamoeba keratitis is always very long and demanding and its management requires adequate experience because it is not always easy to evaluate the response to treatment and complications can be very serious and difficult to manage. Resistance to therapy can also occur during treatment and must be distinguished from drug-induced toxicity.

In the event of deterioration during treatment, any therapy can be stopped and the response observed: if the clinical picture improves, it can be concluded that it was drug toxicity and replace one of the drugs used with an analogue (e.g., PHMB with chlorhexidine or vice versa) or reduce the number of administrations. In the event of failure to improve, it must be concluded that it was resistance; in this regard, you can replace a drug with an analogue (as above) or increase its concentration. $^{22,23,24}$ PHMB is also tolerated at the concentration of $0.04 \%$ and $0.06 \%$.

Although the mechanism of action of PHMB is similar to that of Chlorhexidine, in a case of resistance to both drugs, confirmed by the positivity to culture after repeated sampling, combination of the two drugs may resolve the infection. The effect of the association of the two Biguanides could be the same as that obtained with the increase in the concentration reported above.

With regard to the suspension of treatment, it must be remembered that, unlike bacterial and even fungal infections, the progression of amoebic infection is very slow, so there is no risk in temporarily stopping therapy. The temporary suspension of anti-amoebic drugs has also been proposed as "pulsed" therapy, in 
order to encourage the passage of the parasite from the cystic, more resistant, to the vegetative form, which is much more sensitive to drugs.

In cases where there is no improvement despite all these manouvers, it is advisable to repeat the corneal sampling and proceed to new laboratory tests for Acanthamoeba, bacteria and fungi. Another technique in cases of progressive worsening despite therapy is the conjunctival grafting. It has been shown that blood supply with conjunctival tissue can have a positive effect on both the colliquative necrosis and the infectious component.

Prevention, which always remains of fundamental importance, is practically based on avoiding contact of the corneal lens with contaminated water, since this Acanthamoeba has a ubiquitous diffusion. It is therefore recommended to always avoid the use of corneal contact lenses in the pool or in the shower, not to wash them under running tap water and to frequently replace the relative container of these lenses. ${ }^{8}$

\section{Conflict of Interest}

Authors declared no conflict of interest.

\section{REFERENCES}

1. Puig M, Weiss M, Salinas R, Johnson DA, Kheirkhah A. Etiology and Risk Factors for Infectious Keratitis in South Texas. J Ophthalmic Vis Res. 2020; 15 (2): 128-137. Doi: 10.18502/jovr.v15i2.6729.

2. Nayeri T, Bineshian F, Khoshzaban F, Asl AD, Ghaffarifar F. Evaluation of the effects of Rumex obtusifolius seed and leaf extracts against Acanthamoeba: An in vitro study. Infect Disord Drug Targets, 2020 Apr 22. [Epub ahead of print]. Doi: 10.2174/1871526520666200422111044.

3. Musayeva A, Riedl JC, Schuster AK, WasielicaPoslednik J, Pfeiffer N, Gericke A. Topical Voriconazole as Supplemental Treatment for Acanthamoeba Keratitis. Cornea, 2020 Mar 24.

[Epub ahead of print]. Doi: 10.1097/ICO.0000000000002315.

4. Carnt NA, Subedi D, Lim AW, Lee R, Mistry P, Badenoch PR, et al. Prevalence and seasonal variation of Acanthamoeba in domestic tap water in greater Sydney, Australia. Clin Exp Optom. 2020 Mar 29. [Epub ahead of print]. Doi: 10.1111/cxo.13065.
5. Weinstein I, Fries FN, Szentmáry N, Seitz B, Da. Distinctive Wessely immune ring in keratitis-a chameleon. Ophthalmologe, 2020 Mar 24.

Doi: 10.1007/s00347-020-01084-8.

6. Sudano RA, Asero A. An Original Use of a Bioluminescence Assay to Test the In Vitro Efficacy of Polihexanide in the Eradication of Acanthamoeba Cysts. Cornea, 2020 Mar 13. [Epub ahead of print]. Doi.10.1097/ICO.0000000000002308.

7. Sifaoui I, Capote Yanes EC, Reyes-Batlle M, Rodríguez-Expósito RL, Piñero JE, LorenzoMorales J. Combined Amoebicidal Effect of Atorvastatin and Commercial Eye Drops against Acanthamoeba castellanii Neff: In Vitro Assay Based on Mixture Design. Pathogens. 2020 Mar 17; 9 (3). pii: E219. Doi: 10.3390/pathogens9030219.

8. Hasni I, Andréani J, Colson P, La Scola B. Description of Virulent Factors and Horizontal Gene Transfers of Keratitis-Associated Amoeba Acanthamoeba Triangularis by Genome Analysis. Pathogens, 2020 Mar 16; 9 (3): pii: E217.

Doi: $10.3390 /$ pathogens 9030217.

9. Lang SJ, Böhringer D, Reinhard T. Necrotizing scleritis after acanthamoeba keratitis. Ophthalmologe, 2020 Mar 11. [Epub ahead of print] Doi: 10.1007/s00347-020-01080-y.

10. Carnt NA, Subedi D, Connor S, Kilvington S. The relationship between environmental sources and the susceptibility of Acanthamoeba keratitisin the United Kingdom. PLoS One, 2020 Mar 11; 15 (3): e0229681. Doi: 10.1371/journal.pone.0229681.

11. Peyman A, Pourazizi M, Peyman M, Kianersi F. Natural Honey-Induced Acanthamoeba keratitis. Middle East Afr J Ophthalmol. 2020; 26 (4): 243-245. Doi: 10.4103/meajo.MEAJO_56_18. eCollection 2019 Oct-Dec.

12. Brunet K, Eestermans R, Rodier MH, Cateau E. In vitro activity of isavuconazole against three species of Acanthamoeba. J Fr Ophtalmol. 2020; 43 (4): 330-333. Epub 2020 Mar 6. Doi: 10.1016/j.jfo.2019.06.014.

13. Kot K, Kosik-Bogacka D, Kupnicka P, LanochaArendarczyk N. Antioxidant defense in the eyes of immunocompetent and immunosuppressed mice infected with Acanthamoeba spp. Parasit Vectors, 2020; 13 (1): 123. Doi: 10.1186/s13071-020-3979-5.

14. Raghavan A, Veerappan S, Rangarajan V, Rajaraman R, Salian R, Mk J, et al. Fulminant Acanthamoeba Endophthalmitis After Cataract Surgery-A Case Report. Cornea, 2020 Feb 14. [Epub ahead of print]. Doi: 10.1097/ICO.0000000000002289.

15. Atalay HT, Uysal BS, Sarzhanov F, Usluca S, Yeşilırmak N, Özmen MC, et al. Rose BengalMediated Photodynamic Antimicrobial Treatment of Acanthamoeba Keratitis. Curr Eye Res. 2020 Feb 27: 16. [Epub ahead of print].

Doi: 10.1080/02713683.2020.1731830. 
16. Annapurna NV, Bagga B, Garg P, Joseph J, Sharma S, Kalra P, et al. Management of severe Acanthamoeba keratitis and complicated cataract following laser in situ keratomileusis. Indian $\mathbf{J}$ Ophthalmol. 2020 Mar; 68 (3): 515-516.

Doi: 10.4103/ijo.IJO_492_19.

17. Bagga B, Garg P, Joseph J, Mohamed A, Kalra P. Outcome of therapeutic deep anterior lamellar keratoplasty in advanced Acanthamoeba keratitis. Indian J Ophthalmol. 2020 Mar; 68 (3): 442-446.

Doi: 10.4103/ijo.IJO_307_19.

18. Singh M, Gour A, Gandhi A, Mathur U, Farooqui JH. Demographic details, risk factors, microbiological profile, and clinical outcomes of pediatric infectious keratitis cases in North India. Indian $\mathrm{J}$ Ophthalmol. 2020 ; 68 (3): 434-440.

Doi: 10.4103/ijo.IJO_928_19.

19. Morgan SR, Pilia N, Hewitt M, Moses RL, Moseley $\mathbf{R}$, Lewis PN, et al. Controlled in vitro delivery of voriconazole and diclofenac to the cornea using contact lenses for the treatment of Acanthamoeba keratitis. Int J Pharm. 2020; 579: 119102.

Doi: 10.1016/j.ijpharm.2020.119102. Epub 2020 Jan 31.

20. Burgos-Blasco B, Arriola-Villalobos P, AriñoGutiérrez M, Gegúndez-Fernández JA, Díaz-Valle D. Penetrating keratoplasty with Krumeich ring for corneal leukoma secondary to Acanthamoeba keratitis. J Fr Ophtalmol. 2020;43 (3): e115-e118. Epub 2020 Jan 20. Doi: 10.1016/j.jfo.2019.08.005.
21. Sun Y, Li W, Wang M, Xing Q, Sun X. Clinical diagnosis and treatment of rare painless keratitis caused by three pathogens: clinical practice and experiential discussion. J Int Med Res. 2020; 48 (1): 300060519895671. Doi: 10.1177/0300060519895671.

22. Nielsen SE, Ivarsen A, Hjortdal J. Increasing incidence of Acanthamoeba keratitis in a large tertiary ophthalmology department from year 1994 to 2018. Acta Ophthalmol. 2019 Dec 29.

Doi: 10.1111/aos.14337. [Epub ahead of print].

23. Boukari M, Zhioua-Braham I, Souissi L, Kaouel H, Errais K, Ammous I, et al. Contact lens-related polymicrobial keratitis: Acanthamoeba spp. and Candida albicans. J Fr Ophtalmol. 2020 Jan; 43 (1): e39-e40. Epub 2019 Dec 24.

Doi: 10.1016/j.jfo.2019.10.001.

24. Shi L, Hager T, Fries FN, Daas L, Holbach L, Hofmann-Rummelt C, et al. Reactive uveitis, retinal vasculitis and scleritis as ocular end-stage of Acanthamoeba keratitis: a histological study.Int J Ophthalmol. 2019 Dec 18; 12 (12): 1966-1971. Doi: 10.18240/ijo.2019.12.20. eCollection 2019.

\section{Author's Designation and Contribution}

Mauro Salducci: Professor; Literature review and manuscript writing. 